\title{
Neural Network Based Prediction of Stable Equivalent Series Resistance in Voltage Regulator Characterization
}

\author{
Mohd Hairi Mohd Zaman ${ }^{\text {* }}$, M. Marzuki Mustafa ${ }^{2}$, M. A. Hannan ${ }^{3}$, Aini Hussain ${ }^{4}$ \\ ${ }^{1,2,4}$ Department of Electrical, Electronic and Systems Engineering, Universiti Kebangsaan Malaysia \\ ${ }^{3}$ College of Engineering, Universiti Tenaga Nasional, Malaysia
}

\begin{tabular}{l} 
Article Info \\
\hline Article history: \\
Received Nov 13, 2017 \\
Revised Jan 14, 2018 \\
Accepted Jan 28, 2018 \\
\hline
\end{tabular}

Keywords:

Equivalent series resistance Neural network

Output capacitor

Stable region

Voltage regulator

\begin{abstract}
High demand on voltage regulator (VR) currently requires VR manufacturers to improve their time-to-market, particularly for new product development. To fulfill the output stability requirement, VR manufacturers characterize the VR in terms of the equivalent series resistance (ESR) of the output capacitor because the ESR variation affects the VR output stability. The VR characterization outcome suggests a stable range of ESR, which is indicated in the ESR tunnel graph in the VR datasheet. However, current practice in industry manually characterizes VR, thereby increasing the manufacturing time and cost. Therefore, an efficient method based on multilayer neural network has been developed to obtain the ESR tunnel graph. The results show that this method able to reduce the VR characterization time by approximately 53\% and achieved critical ESR prediction error less than 5\%. This work demonstrated an efficient and effective approach for VR characterization in terms of ESR.
\end{abstract}

Copyright $@ 2018$ Institute of Advanced Engineering and Science. All rights reserved.

\section{Corresponding Author:}

Mohd Hairi Mohd Zaman,

1Department of Electrical, Electronic and Systems Engineering,

Universiti Kebangsaan Malaysia.

Email: hairizaman@ukm.edu.my

\section{INTRODUCTION}

Most electronic products have an important power converter component called voltage regulator (VR). The VR converts the noisy and unstable direct current (DC) input supply (voltage and current) to a stable, constant, accurate, and load-independent DC output supply. The VR has high demand in recent years because of the emerging market of high-performance portable electronic devices with complex and integrated design. Furthermore, new applications, such as system-on-chip and Internet-of-things, require smart devices with tight power consumption specification [1], [2]. All the aforementioned products generally have a short time-to-market. Therefore, VR manufacturers must meet the high market demand without compromising the high-quality requirement [3].

One of the requirement is the VR ability to produce a stable output in various loading conditions. Particularly, VR should quickly react to any disturbances, such as during abrupt load current change, without generating excessive undershoot and overshoot. To ensure stability, a typical VR circuit requires an output capacitor, with optimal internal parasitic resistance, namely equivalent series resistance, ESR. Though the ESR is important to compensate the control loop inside the VR [4], [5], the variations in age and temperature increase the ESR and eventually cause VR output instability [6], [7]. It means that the VR is only stable in a limited ESR range, which is indicated in the datasheet in a special graph called ESR tunnel graph as shown in Figure 1. This ESR tunnel graph indicates the stable and unstable regions for a range of outputs or load currents and the ESR values of the capacitor. The critical points, such as the critical ESR and its corresponding undershoot, reside on the boundary between stable and unstable regions. This stability boundary must conform to the design specification. 


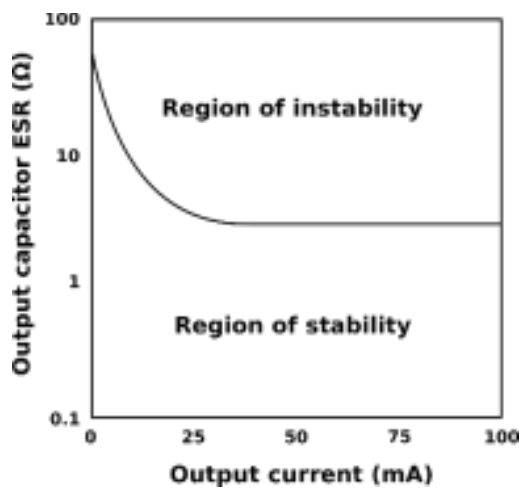

Figure 1. ESR tunnel graph example

Thus, in the VR design and quality assurance phases, it is necessary to investigate and characterize the stable ESR range for all possible load currents. During new product development and manufacturing, VR characterization in terms of ESR is a challenging task because the accurate stability boundary in the ESR tunnel graph is typically unknown. Different VR samples have unique ESR tunnel graphs as a result of variations in the manufacturing process. Since typically no prior mathematical model is provided by the VR manufacturers, the undershoot and overshoot are only accurately measured from the actual VR response during abrupt load current change, where the ESR and load current are iteratively configured [3]-[5]. This iterative process requires a large number of data that should be acquired and analyzed. In addition, current practice in industry manually characterize the VR in terms of stable ESR range. Consequently, current VR characterization takes a lot of time and high cost, due to iterative and manual process.

Clearly, there is a need to speed up the VR characterization. One way to reduce the total characterization time is to limit the number of acquired data. As a consequent, there will be some missing data for certain loading condition. In this case, some questions may arise. For example, how to obtain the undershoot and overshoot for the remaining loading condition with missing data, furthermore no prior model is available? How reliable is the critical ESR extracted from the ESR tunnel graph for this missing data situation? Hence, an efficient and reliable VR characterization method in terms of stable ESR range, with limited acquired data, is required.

Considerable research has been conducted to characterize the VR, but not specific in terms of ESR [8]. Most works in characterizing the VR require knowledge of the internal model of the VR [4], [5]. In most cases, manufacturers do not provide this internal model information. Given that the main challenge in VR characterization is to determine the capacitor ESR, the problem can also be considered as a capacitor condition monitoring (CM) problem, which typically monitors the capacitor characteristics such as capacitance and ESR in power converter application [6], [7], [9]-[15]. Although many works have been conducted, only a few studies have focused on the power converter packaged in integrated circuits. Furthermore, the main purpose is preventive maintenance, not for determining the stable range of capacitor ESR.

Therefore, the black box approach using advanced algorithm based on the data collected during manual VR characterization is a promising research area, as well as artificial intelligence techniques such as neural network $(\mathrm{NN})$ [11]. NN approach is suitable because it does not require VR internal model and external signal injection. Furthermore, the VR terminal voltages and currents are available for dataset development purpose to train the NN structure. The NN-based system design will be less complexity yet reliable. Besides, NN-based approach is widely applied in other engineering applications [16], [17].

\section{RESEARCH METHOD}

The proposed efficient VR characterization in this work predicted the stable range of capacitor ESR using the multilayer NN-based approach. In this work, the NN approach was used with a reduced number of dataset from manual characterization results to achieve efficient VR characterization. The critical ESR values that reside on the stability boundary in the ESR tunnel graph were also predicted. The performance of the proposed method was eventually validated with the manual VR characterization results. The overall process is depicted in Figure 2. The process involves manual VR characterization, critical ESR benchmark search, dataset generation, $\mathrm{NN}$ training, critical ESR prediction using trained $\mathrm{NN}$, and characterization time comparison. 


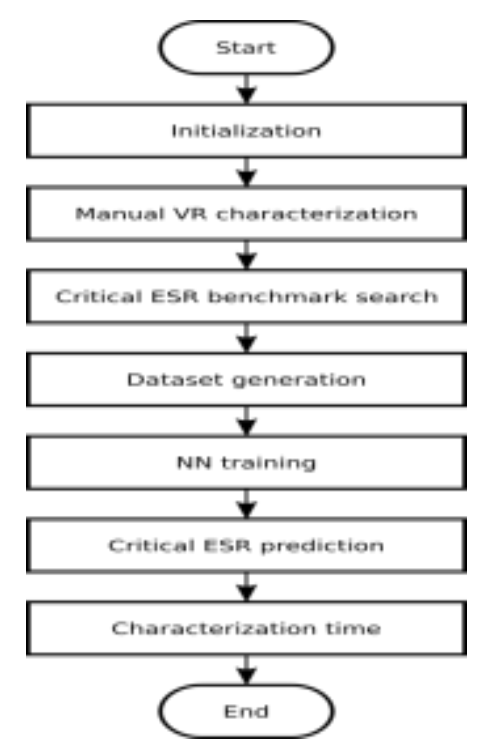

Figure 2. Flowchart of the overall process

\subsection{Multilayer NN}

The multilayer NN used in this work consists of an input layer, a hidden layer, and an output layer [16]. The input layer receives the external input signals and redistributes these signals to the hidden layer. Then, the neuron weights in the hidden layer represent the significant features in the input signals. Finally, the output layer produces the output pattern of multilayer NN.

Therefore, the weight in NN should be adjusted during the NN training phase to reduce the difference between the actual and desired outputs of multilayer NN. Given that many weights should be computed in NN, the back-propagation learning algorithm is used. Input signals are propagated through the network from the input layer to the hidden layer and eventually the output layer. Meanwhile, the error signals are propagated backward, starting from the output layer to the hidden layer.

\subsection{VR Selection}

In this work, LT1963A series VR from Linear Technology was selected because its datasheet has more description and information than those of other VR models manufactured by other VR manufacturers. Furthermore, Linear Technology provides the VR model libraries for most of their products together with the LTspice IV simulation software program, which is available online and can be downloaded from their website.

\subsection{Test Circuit Development}

LT1963A series VR has a number of models depending on its output voltage. One of these models is the adjustable-type VR model used in this work. Figure 3 displays the simplified internal architecture inside the adjustable-type VR in general. Typically, an adjustable-type VR has four terminals, namely, input (IN), output (OUT), adjustable (ADJ) and ground (GND) terminals. This type of VR has an internal series pass element, which is typically a PMOS transistor, an error amplifier, and a voltage reference. The error amplifier function senses any changes in the output voltage measured at the OUT terminal through feedback dividers, $R_{1}$ and $R_{2}$.

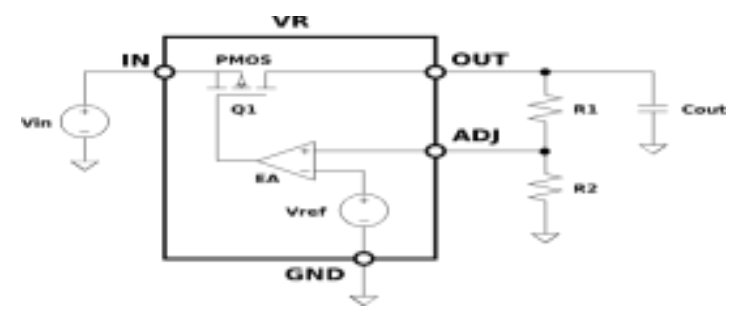

Figure 3. Simplified internal architecture of the adjustable-type VR 
The output voltage of the adjustable-type VR, $\mathrm{V}_{\text {OUT }}$, can be computed by analyzing the circuit in steady-state condition. In steady-state condition, equivalent voltages will be generated at both error amplifier inputs, and consequently, the capacitor will not discharge. Therefore, the steady-state output voltage, $\mathrm{V}_{\text {OUT(SS) }}$, is the summation of voltages across $R_{1}$ and $R_{2}$. The current flowing through $R_{1}$ is approximately equivalent to the current approaching the ADJ terminal, $\mathrm{I}_{\mathrm{ADJ}}$. Meanwhile, $\mathrm{V}_{\mathrm{R} 2}$ is equal to the voltage at the $\mathrm{ADJ}$ terminal, $\mathrm{V}_{\mathrm{ADJ}}$. Using voltage division at the ADJ terminal,

$$
\mathrm{V}_{\mathrm{R}_{2}}=\mathrm{V}_{\mathrm{ADJ}}=\left(\frac{\mathrm{R}_{2}}{\mathrm{R}_{1}+\mathrm{R}_{2}}\right) \mathrm{V}_{\mathrm{OUT}}
$$

Thus, $\mathrm{V}_{\text {OUT(SS) }}$ becomes,

$$
\mathrm{V}_{\text {OUT(SS) }}=\mathrm{I}_{\mathrm{ADJ}} \mathrm{R}_{1}+\left(1+\frac{\mathrm{R}_{1}}{\mathrm{R}_{2}}\right) \mathrm{V}_{\mathrm{ADJ}}
$$

Figure 4 shows the LT1963A test circuit implemented in this work with the output voltage, $\mathrm{V}_{\text {OUT(SS) }}$, can be computed based on (2). As stated in the LT1963A datasheet, $\mathrm{V}_{\mathrm{ADJ}}$ is $1.21 \mathrm{~V}$ and $\mathrm{I}_{\mathrm{ADJ}}$ is $3 \mu \mathrm{A}$ at $25{ }^{\circ} \mathrm{C}$ for the output voltage range between $1.21 \mathrm{~V}$ to $20 \mathrm{~V}$. Therefore, the VR circuit shown in Figure 2 produces $\mathrm{V}_{\text {OUT(SS) }}$ of $4.9691 \mathrm{~V}$. The output voltage tolerance is approximately $3 \%$ over the full operating temperature range. The datasheet also indicates that the suitable output capacitor is $10 \mu \mathrm{F}$ with maximum allowable ESR of $3 \Omega$.

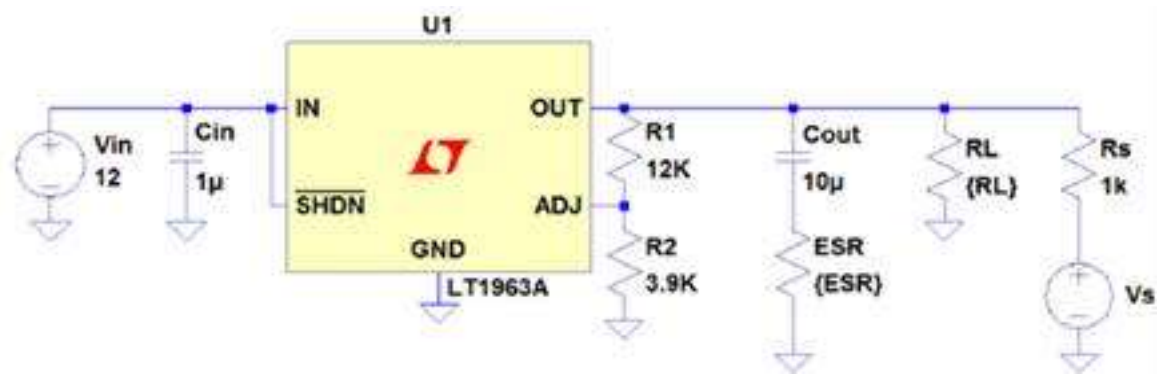

Figure 4. LT1963A VR test circuit

\subsection{Dataset Development}

The dataset developed in this work was initially generated using the LTspice IV software from Linear Technology and processed using the MATLAB software from Mathworks. All further analyses, including dataset development, were executed in MATLAB with Neural Network Toolbox. The information in the dataset includes the input and output voltages and currents, stability condition, minimum and maximum output voltages, and load transient response undershoot and overshoot.

\subsection{Manual VR Characterization}

Prior to the NN-based VR characterization, we conducted manual VR characterization through circuit simulation in LTspice to collect a raw dataset and generate the baseline or benchmark for the subsequent process. The manual process of VR characterization uses the context of changing the load current and ESR values during circuit simulation. These values were manually incremented step by step. In manual VR characterization, the range of load current, $\mathrm{I}_{\mathrm{OUT}}$, is from $0.1 \mathrm{~A}$ to $0.5 \mathrm{~A}$, with $0.05 \mathrm{~A}$ increment. Meanwhile, the range of ESR is from $5 \Omega$ to $100 \Omega$, with $5 \Omega$ increment.

Manual VR characterization was started by initializing the input voltage, $V_{\text {IN }}$. Once the start time of characterization was recorded and the dataset was initialized, the VR circuit was simulated in LTspice for each $\mathrm{I}_{\text {OUT }}$ and ESR. Circuit simulation in LTspice was fully controlled by MATLAB. In detail, a MATLAB program was developed to generate the circuit netlist file in the text file form. Circuit netlist file contains all circuit nodes and connection information, as well as component values such as capacitor's capacitance and ESR. Then MATLAB is used to run the LTspice software to simulate the generated circuit netlist file. After LTspice simulation was completed, the circuit raw data was imported into MATLAB for further processing.

In detail, after waiting for $1.5 \mathrm{~s}, \mathrm{I}_{\mathrm{OUT}}$ was disturbed or changed abruptly by energizing the signal generator, $\mathrm{V}_{\mathrm{s}}$, before stopping the simulation $1.5 \mathrm{~s}$ later. Simultaneously, the VR output response comprising

\footnotetext{
Neural Network Based Prediction of Stable Equivalent Series Resistance in... (Mohd Hairi Mohd Zaman)
} 
output voltage, $\mathrm{V}_{\mathrm{OUT}}$, and outpur current, $\mathrm{I}_{\mathrm{OUT}}$, were acquired. Then, the minimum and maximum output voltages, $\mathrm{V}_{\text {OUT }_{\text {min }}}$ and $\mathrm{V}_{\text {OUT }_{\max }}$, were also obtained. After that, the output voltage undershoot, $\mathrm{V}_{\mathrm{US}}$, and the RMS values of input and output voltages and currents $\left(\mathrm{V}_{\mathrm{IN}_{\mathrm{RMS}}}, \mathrm{V}_{\mathrm{OUT}_{\mathrm{RMS}}}, \mathrm{I}_{\mathrm{IN}_{\mathrm{RMS}}}, \mathrm{I}_{\mathrm{OUT}_{\mathrm{RMS}}}\right)$ were computed. These values will be used as NN inputs during NN training in NN-based VR characterization.

For each combination of $\mathrm{I}_{\text {OUT }}$ and ESR value, the VR stability was determined. The VR stability was judged by checking whether the computed undershoot and overshoot are within the output voltage tolerance or not. However, practically, judging whether $\mathrm{V}_{\text {OUT }_{\min }}$ and $\mathrm{V}_{\text {OUT }_{\max }}$ are within the output voltage tolerance is sufficient to conclude that the VR is stable or unstable. Afterward, the ESR tunnel graph was plotted for each combination of $\mathrm{I}_{\text {OUT }}$ and ESR together with its stability condition. The critical ESR values in manual VR characterization were determined for each $\mathrm{I}_{\text {OUT }}$. After all $\mathrm{I}_{\text {OUT }}$ and ESR values have been characterized, the dataset was updated. Finally, the end time of characterization was recorded, and the total time of characterization was computed.

\subsection{NN Training}

This work applied NN in predicting the critical ESR to reduce the VR characterization time. The most important process is to train the NN structure and find the best configuration (inputs, training function and number of hidden nodes). In addition, the optimum number of datasets will also be determined. In this work, the NN target was the output voltage undershoot and the NN inputs were load current, ESR and other inputs as shown in Figure 5. Other inputs are based on the NN input combination options depicted in Table 1. These NN input combination were required to find the most significant features in terms of VR terminal voltages and currents that able to increase the NN prediction accuracy. For all NN input combination, four different number of hidden nodes were used, namely, 5, 10, 15 and 20 nodes. In addition, there were total 180 data points in the original manual characterization dataset. $70 \%$ of the dataset was used for NN training. Meanwhile, $15 \%$ of the dataset was utilized for validation and $15 \%$ of the dataset was used for testing the trained NN structure.

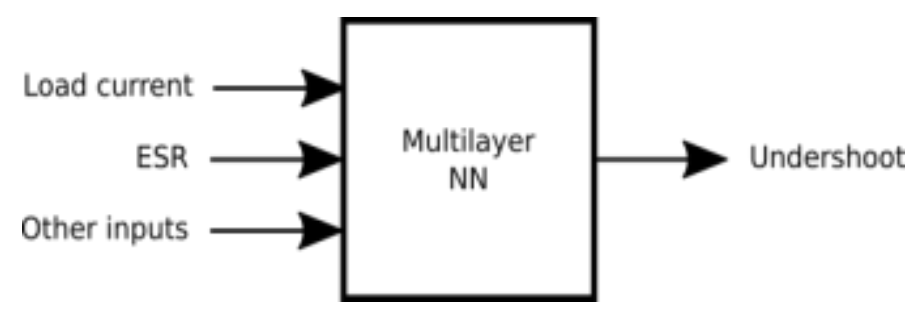

Figure 5. Block diagram of NN-based VR characterization

Table 1. NN input combination options

\begin{tabular}{cl}
\hline Option & Input combination \\
\hline 1 & Initial load current, and ESR \\
2 & Initial load current, ESR, and RMS output current \\
3 & Initial load current, ESR, and RMS output voltage \\
4 & Initial load current, ESR, and RMS input current \\
5 & Initial load current, ESR, and RMS input voltage \\
6 & Initial load current, ESR, RMS input current, and RMS output voltage \\
7 & Initial load current, ESR, RMS input current, and RMS input voltage \\
8 & Initial load current, ESR, RMS input current, RMS input voltage, RMS \\
& output current, and RMS output voltage \\
\hline
\end{tabular}

The first step in NN-based VR characterization is similar to that in manual characterization, which is to record the start time of characterization. Then, the manual characterization dataset was imported into MATLAB. After training the $\mathrm{NN}$ with different configurations ( $\mathrm{NN}$ inputs, training function and number of hidden nodes) and a dataset reduction factor, the best NN configuration was determined based on the minimum prediction error. For each configuration, NN was trained using a reduced number of manual VR characterization datasets. Simultaneously, the undershoot prediction performance metrics obtained for every configuration was computed. This process was repeated 30 times. 


\subsection{Critical ESR Prediction}

The best configuration values were then used to train back NN. After completing the NN training phase, the critical ESR for each load current was predicted. However, based on Table 1, options no. 2 to 8 include other inputs, such as unknown RMS values of the input and output of currents and voltages. Therefore, these RMS values should be interpolated first. The critical ESR prediction started by determining the initial critical ESR based on the outcome of the trained NN. Then, refined ESR increment was implemented to produce a new set of critical ESR candidates based on the initial critical ESR range. Subsequently, the trained NN was used to predict the undershoot value for each load current and critical ESR candidate together with another interpolated input, if any exists. Afterward, the VR stability was determined. The last critical ESR candidate that produces a stable condition was selected as the critical ESR for that particular load current. Then, the ESR tunnel graph for NN-based VR characterization was plotted. Finally, the stop time of characterization was recorded, and the total time of characterization was computed.

\section{RESULTS AND ANALYSIS}

\subsection{Manual VR Characterization Results}

The main aim of this work is to investigate an efficient method to characterize VR in terms of stable ESR range. In other words, the purpose is to find the critical ESR values that lie on the stability boundary in the ESR tunnel graph. Figure 6(a) shows the ESR tunnel graph obtained from manual VR characterization, which also serves as the benchmark. For each load current and ESR, the circle mark represents the stable VR output, whereas the cross mark indicates instability. A clear boundary exists between stable and unstable regions at low load current and high ESR. In detail, the undershoot values in the unstable region are higher than those in the stable region. This undershoot distribution is depicted in Figure 6(b).

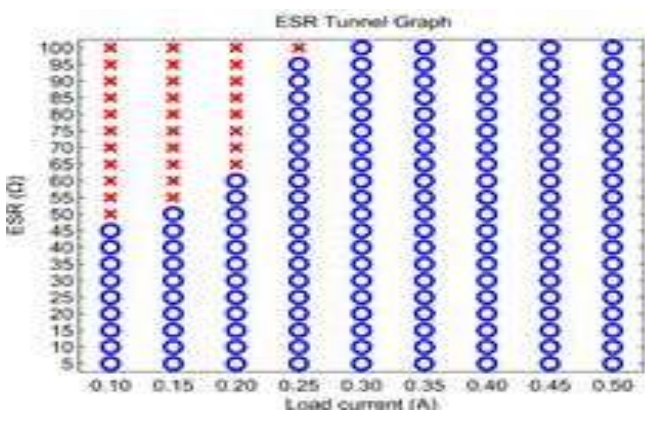

(a)

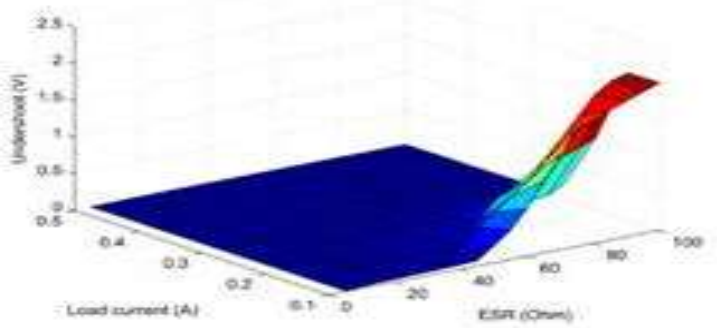

(b)

Figure 6. (a) ESR tunnel graph of manual VR characterization (b) Undershoot distribution

Furthermore, the outcome of manual VR characterization indicates that each load current has a unique critical ESR. For example, Figure 6(a) shows that the critical ESR for a load current of 0.1 A resides between $45 \Omega$ and $50 \Omega$, which is the critical ESR range for that particular load current. Then, the ESR values were refined in this critical ESR range to determine the actual critical ESR for each load current. In particular, the ESR was refined with $1 \Omega$ increment compared with $5 \Omega$ in the initial manual VR characterization.

\subsection{NN-based VR Characterization Results}

After completing manual VR characterization, the NN-based VR characterization was conducted. All VR output undershoot values were predicted using the trained NN with a reduced number of datasets. The training dataset was originally extracted from the results of manual VR characterization. Figure 7(a) displays the ESR tunnel graph of NN-based VR characterization, which has a similar pattern to that of manual VR characterization, as depicted in Figure 6(a). The most important outcome is the predicted critical ESR based on the NN approach. For example, Figure 7(a) depicts that the critical ESR for a load current of $0.1 \mathrm{~A}$ is located between $45 \Omega$ and $50 \Omega$. This critical ESR range is equivalent to that in manual VR characterization shown in Figure 6(a). The predicted ESR for a load current of 0.1 A is $46 \Omega$ instead of $47 \Omega$ in manual characterization. Subsequently, this ESR value was simulated in LTspice for undershoot verification purpose. The LTspice simulation result shows that, the undershoot is $0.1012 \mathrm{~V}$. Meanwhile, the predicted undershoot in NN-based VR characterization is $0.1199 \mathrm{~V}$. Thus, both undershoot values are similar and acceptable.

\footnotetext{
Neural Network Based Prediction of Stable Equivalent Series Resistance in... (Mohd Hairi Mohd Zaman)
} 


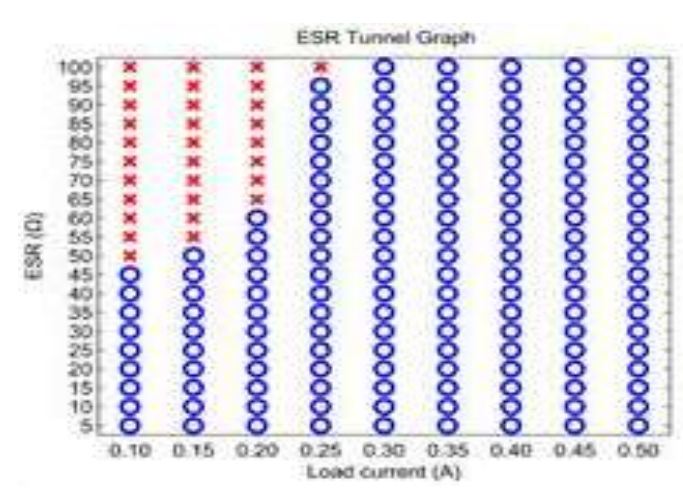

(a)

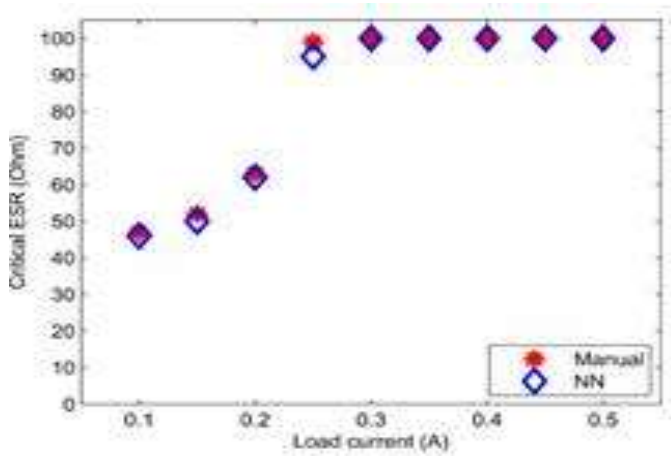

(b)

Figure 7. (a) ESR tunnel graph of NN-based VR characterization (b) Critical ESR comparison between manual and NN-based VR characterization

In addition, Figure 7(b) shows the critical ESR comparison between manual and NN-based VR characterization for a dataset reduction factor of 1 . In this work, five performance metrics were used to evaluate the predicted critical ESR performance, namely mean squared error (MSE), root mean squared error (RMSE), mean absolute error (MAE), correlation coefficient (R2) and relative error (RE) based on the following equations,

$$
\begin{aligned}
& \text { MSE }=\frac{1}{N} \sum_{i=1}^{N}\left[y(i)-y_{p}(i)\right]^{2} \\
& \text { RMSE }=\sqrt{\frac{1}{N} \sum_{i=1}^{N}\left[y(i)-y_{p}(i)\right]^{2}} \\
& \text { MAE }=\frac{1}{N} \sum_{i=1}^{N}\left|y(i)-y_{p}(i)\right| \\
& R^{2}=\frac{\sum_{i=1}^{N}\left[(y(i)-\bar{y}(i))\left(y_{p}(i)-\overline{y_{p}}(i)\right)\right]}{\sqrt{\sum_{i=1}^{N}[y(i)-\bar{y}(i)]^{2} \sum_{i=1}^{N}\left[y_{p}(i)-\overline{y_{p}}(i)\right]^{2}}} \\
& \text { RE }=\left(\frac{y(i)-y_{p}(i)}{y(i)}\right) \times 100
\end{aligned}
$$

where $\mathrm{y}$ and yp are the actual and predicted critical ESR, $\mathrm{N}$ is the number of observations and $\mathrm{i}$ is the load current instant. The computed MSE, RMSE and MAE are 2.4444, 1.5635 and 0.8889, respectively. Meanwhile, the computed R2 is 0.9985 which is close to one and it indicates good critical ESR prediction. Furthermore, RE, which measures the relative accuracy, has a mean value of $1.2891 \%$, with maximum RE of $4.0404 \%$ for a load current of $0.25 \mathrm{~A}$. The maximum RE is set to $5 \%$ in this work. Based on these outcomes, the output capacitor ESR must be less than $46 \Omega$ to ensure a stable VR circuit operation.

\subsection{Best NN Configuration Results}

Critical ESR prediction results with good accuracy were obtained after defining the best configuration of NN structure and dataset reduction factor. Firstly, the overall data distribution based on the NN training function was analyzed. Three types of NN training function options were used in this work; namely, Bayesian regularization (BR), Levenberg-Marquardt and scaled conjugate gradient backpropagation functions. BR training function was selected since it has the lowest MSE of undershoot prediction at 0.99 x10-3. Next, the best $\mathrm{NN}$ input combination was analyzed. Input combination option 3 produced the minimum MSE at $3.52 \times 10-3$. Then, the best number of hidden nodes was also selected among 5, 10, 15 and 20 nodes. In this work, 15 hidden nodes generated the smallest MSE of 6.11 x 10-3. In addition, the best dataset reduction factor among factor of 1,2 or 3, was selected with factor of 1 generated the smallest MSE at $7.26 \times 10-3$. Afterward, the maximum dataset reduction factor was inspected based on the critical ESR prediction error for factor of 1, 2, 3 and 4, where factor of 3 was the best configuration. This result indicates that NN-based VR characterization reduced the data that are required to be manually acquired up to $53 \%$. 


\subsection{Characterization Time Comparison}

The total characterization time was also compared. The total manual characterization time, $\mathrm{t}_{\text {manual }}$ was the total time taken to complete characterizing the VR for all combination of $\mathrm{I}_{\mathrm{OUT}}$ and ESR values. In detail, the time taken for each combination of $\mathrm{I}_{\text {OUT }}$ and ESR including the process to run both MATLAB and LTspice, starting from the circuit netlist file generation until plotting the ESR tunnel graph and determining the critical ESR values. Meanwhile, the total $N N$-based characterization time, $t_{N N-\text { based }}$ included the time taken to generate the reduced number of manual characterization datasets. Furthermore, $t_{\mathrm{NN} \text {-based }}$ also comprises of the time taken to complete all subsequent processes including the NN training, VR output voltage undershoot estimation based on the trained NN structure, VR stability judgement, ESR tunnel graph plotting and finally critical ESR determination. The total characterization time reduction, $t_{\text {reduction }}$ was computed as follows,

$$
\mathrm{t}_{\text {reduction }}=\left(\frac{\mathrm{t}_{\text {manual }}-\mathrm{t}_{\mathrm{NN}-\text { based }}}{\mathrm{t}_{\text {manual }}}\right) \times 100
$$

In this work, the manual characterization process took $4,832 \mathrm{~s}$ to complete, while NN-based approach only needs $2,293 \mathrm{~s}$ to produce a similar result. Therefore, the total characterization time was reduced by $52.6 \%$ and furthermore indicates that the NN-based approach was an efficient VR characterization technique.

\section{CONCLUSION}

An efficient method for predicting the critical ESR and its corresponding undershoot using NN during VR characterization was investigated in this work. This work has developed a method using multilayer NN approach to predict the undershoot and subsequently estimate the stable ESR range in the ESR tunnel graph. Based on the predicted stable ESR range, the critical ESR for each load current has been predicted with good accuracy, even with reduced number of dataset. All results obtained show that the NNbased approach is an efficient and effective VR characterization in terms of ESR of output capacitor.

\section{ACKNOWLEDGEMENTS}

The authors acknowledge the financial support received from Universiti Kebangsaan Malaysia through the research grant no. INDUSTRI-2013-008.

\section{References}

[1] Garimella A, Furth PM. A 1.21V, 100mA, 0.1 $\mu$ F-10 $\mu$ F Output Capacitor Low Drop-Out Voltage Regulator for SoC Applications. Proceedings of the 16th IEEE International Conference on Electronics, Circuits, and Systems (ICECS). Yasmine Hammamet. 2009; 375-378.

[2] Bilberry CC, Mazzola MS, Gafford J. Power Supply on Chip (PwrSoC) Model Identification Using Black-Box Modeling Techniques. Proceedings of the Twenty-Seventh Annual IEEE Applied Power Electronics Conference and Exposition (APEC). Orlando. 2012; 1821-1825.

[3] Musa AH, Zaman MHM, Mohamed R, Mustafa MM. Characterization of Voltage Regulators by Automated Equivalent Series Resistance. Proceedings of the IEEE Conference on Systems, Process and Control (ICSPC). Kuala Lumpur. 2014; 68-72.

[4] Chen KH. Power Management Techniques for Integrated Circuit Design. Singapore: John Wiley \& Sons. 2016.

[5] Rincon-Mora GA. Analog IC Design with Low-Dropout Regulators. Second Edition. United States of America: McGraw-Hill. 2014.

[6] Yang S, Xiang D, Bryant A, Mawby P, Ran L, Tavner P. Condition Monitoring for Device Reliability in Power Electronic Converters: A Review. IEEE Transactions on Power Electronics. 2010; 25(11): 2734-2752.

[7] Wang H, Blaabjerg F. Reliability of Capacitors for DC-Link Applications in Power Electronic Converters: An Overview. IEEE Transactions on Industry Applications. 2014; 50(5): 3569-3578.

[8] Hiremath ASC, Nanda BBY, Gemson C. Characterization of Low Dropout Regulator (LDO). Proceedings of the 1st International Conference on Signal Processing, Image Processing and VLSI (ICrtSIV). Bangalore. 2014; 121 128.

[9] Buiatti GM, Martin-Ramos JA, Garcia CHR, Amaral AMR, Cardoso AJM. An Online and Noninvasive Technique for the Condition Monitoring of Capacitors in Boost Converters. IEEE Transactions on Instrumentation and Measurement. 2010; 59(8): 2134-2143.

[10] Abo-Khalil AG, Lee DC. DC-Link Capacitance Estimation in AC/DC/AC PWM Converters Using Voltage Injection. IEEE Transactions on Industry Applications. 2008; 44(5): 1631-1637.

[11] Soliman H, Wang H, Gadalla B, Blaabjerg F. Condition Monitoring for DC-Link Capacitors Based on Artificial Neural Network Algorithm. Proceedings of the IEEE International Conference on Power Engineering, Energy and Electrical Drives (POWERENG). Riga. 2015; 587-591.

Neural Network Based Prediction of Stable Equivalent Series Resistance in ... (Mohd Hairi Mohd Zaman) 
[12] Soliman H, Wang H, Blaabjerg F. A Review of the Condition Monitoring of Capacitors in Power Electronic Converters. IEEE Transactions on Industry Applications. 2016; 52(6): 4976-4989.

[13] Wechsler A, Mecrow B, Atkinson D, Bennett J, Benarous M. Condition Monitoring of DC Link Capacitors in Aerospace Drives. IEEE Transactions on Industry Applications. 2012; 48(6): 1866-1874.

[14] Wang G, Guan Y, Zhang J, Wu L, Zheng X, Pan W. ESR Estimation Method for DC-DC Converters Based on Improved EMD Algorithm. Proceedings of the IEEE Conference on Prognostics and System Health Management (PHM). Beijing. 2012; 1-6.

[15] Buiatti GM, Amaral AMR, Cardoso AJM. A Very Simple Experimental Technique for Characterising and Diagnosing Electrolytic Capacitors. International Journal of Electrical Engineering Education. 2012; 48(11): 1733.

[16] Jensimiriam E, Seenichamy P, Ambalavanan S. Prediction of Lead-Acid Battery Performance Parameter: An Neural Network Approach. Bulletin of Electrical Engineering and Informatics (BEEI). 2013; 2(1): 65-74.

[17] Khoei HR, Zolfaghari M. New Model Reference Adaptive System Speed Observer for Field-Oriented Control Induction Motor Drives Using Neural Networks. Bulletin of Electrical Engineering and Informatics (BEEI). 2016; $5(1)$. 